\title{
Liderazgo transformacional para consolidar la responsabilidad social en instituciones educativas $^{8}$
}

\author{
Lucía Magdalena Rojas Hernández \\ Doctora en Ciencias Gerenciales \\ Universidad del Sinu - Elias Bechara Zainúm. Colombia \\ Correo electrónico: luciamrojash@gmail.com
}

Maria Eunice Di Fiore Subero

Doctora en Ciencias Gerenciales Universidad Nacional Experimental Rafael Maria Baralt. Venezuela Correo electrónico: mdifiore1966@gmail.com
Recibido: 07/03/2019

Evaluado: $10 / 06 / 2019$

Aceptado: 29/08/2019

\section{Resumen}

El estudio tuvo como objetivo analizar el liderazgo transformacional como herramienta para la responsabilidad social en Instituciones Educativas del Municipio de San Juan del Cesar. La investigación fue analítica, con diseño de campo, no experimental, transeccional. La población estuvo conformada por 23 directivos, 109 docentes y 15 miembros de la comunidad. Se diseñaron tres cuestionarios de 38, 20 y 20 ítems, con escala de cinco opciones de respuesta, a saber: siempre, casi siempre, a veces, casi nunca y nunca. La validez de los instrumentos se determinó con juicio de expertos y análisis discriminante. La confiabilidad con el método de las dos mitades y la corrección de Spearman-Brown, arrojando un valor de .71, .78 y .83. El procesamiento fue mediante estadística descriptiva. El resultado indicó una moderada aplicación del liderazgo transformacional dentro del contexto laboral, lo cual no ha permitido que se convierta en una herramienta para la puesta en marcha de la responsabilidad social.

Filosofía del Liderazgo, Liderazgo Transformacional, Responsabilidad Social - Instituciones Públicas

8 Para citar este artículo: Rojas H., L. y Di Fiore S., M. (2021). Liderazgo transformacional para consolidar la responsabilidad social en instituciones educativas. Informes Psicológicos, 21(1), 117-131 http://dx.doi.org/10.18566/infpsic.v21n1a08 


\section{Transformational leadership to consolidate social responsibility in educational institutions}

Abstract

The study aimed to analyze transformational leadership as a tool for social responsibility in educational institutions of the Municipality of San Juan del Cesar. The research was analytical, with a field, non-experimental, transectional design. The population was made up of 23 directors, 109 teachers and 15 community members. Three questionnaires of 38, 20 and 20 items were designed, with a scale of five response options (always, almost always, sometimes, almost never and never). The validity of the instruments was determined with expert judgment and discriminant analysis. Reliability was measured with the two halves method and Spearman-Brown correction, yielding values of .71, .78 and .83. The processing was done using descriptive statistics. The result indicated a moderate application of transformational leadership within the work context, which has not allowed it to become a tool for the implementation of social responsibility.

Keywords

\section{Liderança transformacional para consolidar a responsabilidade social nas instituições de ensino Resumo}

0 estudo teve como objetivo analisar a liderança transformacional como ferramenta de responsabilidade social em Instituições de Ensino do Município de San Juan del Cesar. A pesquisa foi analítica, com delineamento de campo, não experimental, transversal. A população da amostra foi composta por 23 gestores, 109 professores e 15 membros da comunidade. Foram elaborados três questionários de 38, 20 e 20 itens; com uma escala de cinco opções de resposta; a saber: sempre, quase sempre, às vezes, quase nunca e nunca. A validade dos instrumentos foi determinada com julgamento de especialistas e análise discriminante. Confiabilidade com 0 método das duas metades e a correção de Spearman-Brown, resultando em um valor de .71, .78 e .83. Processamento por meio de estatística descritiva. 0 resultado indicou uma aplicação moderada da liderança transformacional no contexto de trabalho, o que não tem permitido que se torne em uma ferramenta para a implementação da responsabilidade social.

Palavras chave

Filosofia de liderança, liderança transformacional, responsabilidade social, instituições públicas. 


\section{ntroducción}

En un mundo altamente dinámico, los cambios suceden de manera rápida y constante. Sin embargo, la resistencia que se hace al mismo es algo evidente, puesto que la naturaleza humana prefiere lo conocido, lo estable, la costumbre establecida y la satisfacción de seguir haciendo lo mismo, antes que el cambio; el cual requiere de una visión, un destino y un horizonte. El cambio es una ideología.

En ese sentido, no puede obviarse el hecho de que actualmente los cambios son más rápidos y bruscos, debido al incremento de competencias, desarrollos tecnológicos, fenómenos económicos, efectos inflacionarios e internacionalización de las actividades convertidas en una constante ineludible para las organizaciones. Por lo tanto, se requieren procesos gerenciales, administrativos y operativos más eficientes, que influyan de manera definitiva en los resultados, los cuales tendrán que enfrentarse para impulsar el cambio hacia nuevas formas de ser más competitivos y productivos.

\section{Una aproximación hacia el Liderazgo Transformacional}

El concepto de Liderazgo Transformacional está ineludiblemente asociado al aprendizaje organizacional o, tal como lo dice Salazar (2006), "organizaciones educativas que aprenden" (p. 3), ya que el liderazgo educativo en este tiempo se debe caracterizar por la capacidad de aprendizaje de sus líderes y de sus miembros.

El liderazgo, en cualquier contexto, implica un proceso constante de toma de decisiones, donde, tal y como lo plantea Arroyo (2017), no se delega la responsabilidad, pero sí la autoridad. Este aspecto requiere que el líder desarrolle competencias comunicativas para que los miembros de la organización se sientan capaces y motivados a efectuar las acciones en beneficio de la institución.

Desde esta perspectiva, las organizaciones educativas de América Latina y, en el caso particular de Colombia, tienen grandes desafíos. Sin embargo, alcanzar el éxito sólo será posible teniendo dirigentes proactivos, con criterios claros y dispuestos a trabajar guiando los esfuerzos del contingente humano de la organización, hacia la consecución de los objetivos propuestos, así como lograr un desarrollo sostenido de sus capacidades competitivas, tan necesarias en estos momentos de cambios continuos.

Con relación a lo anterior, las nuevas tendencias de las disciplinas que se relacionan con la educación, sociología de la educación, planificación y teorías organizacionales, centran su atención en el nivel micro, esto es, en el nivel operativo del sistema educativo. De allí que el gerente se vea en la necesidad de "desaprender" actitudes que por muchos años se consideraron deseables, antes de abordar los nuevos enfoques de la gestión organizacional y transformar la escuela en un centro de interacción constructiva, de negociación y de exploración; todo ello con la meta de mejorar la calidad educativa. 
En los términos de la investigación realizada, la gestión gerencial representa una función dentro de la escuela, con una importancia fundamental y que genera repercusiones transcendentales a nivel de productividad en esas organizaciones y en la calidad del servicio que ellas prestan.

La sociedad actual, ante su disposición de avance, conjuga posibilidades de éxito a intensa velocidad, como consecuencia de los constantes cambios políticos, económicos, sociales e incluso culturales; generados por un mundo influido por un fenómeno de importancia mundial, como lo es el de la globalización. Ante este hecho, los avances en las tecnologías de información y comunicación hacen que se produzca una revolución, y los adelantos científicos justifican la necesidad de generar nuevos conocimientos, llevando a los individuos a tomar decisiones que pudieran ser acertadas o desacertadas, pero forman parte de la complejidad misma del devenir del entorno en el cual se desenvuelven.

En este sentido, Suárez (2019) efectuó un estudio sobre la relación entre el liderazgo transformacional y la productividad en los docentes de la escuela fiscal Antonio José de Sucre de la provincia de Guayas, Ecuador, evidenciándose una relación positiva entre ambas variables.

El liderazgo transformacional, para que sea efectivo, debe ser una cualidad personal del líder, en este caso, de la institución educativa; esta visión se corresponde con la concepción que plantea Arroyo (2017) quien hace un análisis de la figura del líder como un factor que le otorga seguridad desde el punto de vista psicológico a los miembros de la organización.
Dentro de ese contexto están inmersas las organizaciones colombianas, más aún cuando se observa cómo evolucionan ciertas condiciones, los cuales podrían desarrollar un fenómeno nominado por especialistas, como "una crisis en todos sus estratos". En este sentido, Piñango (2012) manifiesta, con respecto a la realidad expresada, que es consecuencia de una creciente volatilidad a nivel gerencial, basada en variables exógenas. Esta posición podría entenderse en cuanto a las carencias de consenso nacional, en lo que respecta al país que se debería construir, aunado a la falta de una visión clara y el rumbo a seguir, para el crecimiento de sus organizaciones.

No obstante, el autor citado alude a que los cambios presentes han pasado desde un proteccionismo interno y agobiante intervencionismo estatal, a una apertura indiscriminada, aunada a un liberalismo, donde se omitió la necesidad de regular la economía. De acuerdo a esto, básicamente desde hace más de ocho años, se han venido asumiendo conductas políticas, retomándose la intervención estatista en reglones de comercialización de alimentos, de organizaciones privadas ante la imagen de "nacionalización", y una carente especificidad jurídica para las organizaciones.

Desde esa perspectiva, la situación descrita se puede entender como una restricción al crecimiento económico del país, afectando el adelanto competitivo y limitando a las organizaciones en su funcionamiento. Vale considerar entonces la afirmación emitida por el autor citado, quien, dentro de una crítica reflexiva, expresa que la crisis tanto económica, como social, política y cultural, promete alcanzar niveles de deterioros inéditos, de 
no asumir reformas fundamentales. Las mismas deberán orientarse más allá de la economía, empezando por el sistema político, la conformación del Estado, la práctica de la justicia, el respeto a controles y el estímulo al desarrollo del mercado.

\section{Retos de las instituciones educativas}

En ese sentido, las organizaciones colombianas tienen una meta específica: sobrevivir ante un estado de incertidumbre, requiriendo la participación de líderes que asuman los cambios y estén dentro del campo de la prospectiva, a fin de obtener la excelencia en escenarios extraordinariamente cambiantes y muy complejos.

Las organizaciones educativas cada vez están demandando la presencia de líderes con un liderazgo basado en la autoridad y no en el poder, es decir, un liderazgo transformacional. En este sentido, vale la pena destacar el aporte hecho por Fajardo, Zutta, Caicedo y Luna (2018), quienes estudiaron las variables asociadas al manejo de la autoridad en los docentes. Los docentes con manejo efectivo de la autoridad utilizan las 4 funciones del poder: Prescripción, Regulación, Supervisión y Administración, así como las características de la autoridad, a saber: afinidad y carisma.

En consecución a lo expresado, Dubrir (1913) comenta respecto de la presencia de los líderes organizacionales, que los mismos deberían ser capaces de inspirar, persuadir, influir y motivar, logrando cambios útiles. Entonces, alcanzar un cambio es una meta importante del liderazgo, porque la mayor parte del mejoramiento requiere partir del statu quo. Un líder crea una visión para los demás, y luego dirigir a todos hacia el logro.

La percepción anterior puntualiza la participación de los líderes, quienes, al reconocer la realidad, deberán estar decididos a involucrarse, reconocer el entorno y, ante las circunstancias adversas, desear competir, basándose en sus principios éticos, además de los conocimientos organizacionales, a fin de transformar y poder hacer de sus organizaciones altamente estables, competitivas y sostenibles. Se entiende con ello, la necesidad de generar un ambiente de colaboración y alianzas, donde se permita multiplicar las inteligencias, así como los aportes de todo el personal.

El desarrollo de esa visión dará forma a los valores de la organización, desplazando a ese líder tradicional, que, por creerse poseedor de la verdad, sólo logra conformar un equipo atrincherado en su comodidad, incapaz de asumir riesgos para desarrollarse.

Los retos del liderazgo para los años por venir están en aprender a balancear las maravillas materiales de la tecnología, sin dejar de considerar al talento humano, dada la existencia preocupante de los constantes cambios de las organizaciones, hacia la despersonalización y la virtualización, en busca de una eficiencia mecanicista impulsada por la velocidad del desarrollo tecnológico. Esto convierte a esas organizaciones en autocráticas, y quienes han trabajado en ellas (líderes), se han podido dar cuenta de que ese no es un modelo sostenible. 
Por lo tanto, la posición del liderazgo a través de su líder es asegurar la aprehensión de las organizaciones por conocerse a sí mismas, donde el líder, quien es como un espejo del proceso, conoce las competencias, a los consumidores, al personal y las razones por las que se está en el negocio. Es por ello que, dentro de las instituciones educativas, existe la necesidad del liderazgo para buscar el éxito responsable de formar estudiantes con valores, por lo que Maestres (2016) señala un conjunto de acciones a considerar, en función de lograr los objetivos planteados:

- Crear una filosofía de liderazgo sustentada en la persona. Por lo que se deberá tomar conciencia respecto de esa filosofía, trascendiendo los propios diagramas $u$ organigramas organizacionales, para trazar un camino donde las personas sean tomadas en cuenta y adquieran el deseo de participar.

- Entender que el liderazgo ocurre en comunidades de conocimiento. La sociedad del conocimiento, de acuerdo con lo dicho por Drucker (1998), se vislumbra como un ambiente competitivo para las organizaciones y para las personas, por cuanto, mientras mayor sea la información que se genera, mayor es la necesidad de convertirla en conocimiento.

- Desarrollar estrategias con visión de largo plazo. Porter y Kramer (2002) señalan que "en el presente siglo una organización exitosa necesita líderes con la fuerza requerida para delinear una estrategia y luego entusiasmar a los demás en el proceso de llevarla a la práctica” (p. 50).

- Lograr compromiso grupal para el manejo del negocio. Los líderes más exitosos serán los que puedan identificarse mejor con las áreas de potencial de la gente, y lograr desarrollar arreglos permitiendo obtener la máxima colaboración posible.

- Capacidad para asumir grandes riesgos e intentar cosas nuevas. Los líderes del nuevo siglo tendrán que desarrollar su capacidad para desenvolverse en ambientes inciertos, con elevados costos de oportunidad, en los cuales la certeza y la comodidad de mercados garantizados, han sido bruscamente sustituidos por cambios profundos en la situación competitiva y reacomodos empresariales con intención de garantizar el cambio permanente.

- Visión de liderazgo social. Este punto debe ser tratado desde el punto de vista ético. Para ello, la visión del líder debe estar enmarcada en este postulado: La organización ha de participar con la comunidad en la solución de los problemas. La responsabilidad del líder consiste no solo en gestionar programas sociales con las comunidades, sino en educar a sus colaboradores en esta filosofía, para que se convierta en una gestión común en toda la organización.

- Tolerancia y capacidad para desenvolverse en un ambiente de incertidumbre sociopolítica. 
- Habilidades negociadoras para el manejo de una nueva realidad sindical.

En tal sentido, el tener éxito organizacional depende de aprender y seguir aprendiendo, innovar y estar en el perpetuo proceso de creación y adaptación a las cambiantes circunstancias del entorno.

Es importante mencionar que el éxito o el fracaso de cualquier organización, empresa o sociedad, es algo que está determinado por la calidad del líder y en todos los casos los líderes son los responsables de hacer que cualquier organización funcione adecuadamente y con éxito. Actualmente ha quedado atrás la era industrial, y se ha entrado a la era del conocimiento, donde la economía mundial gira a su entorno. Hay que tener cuidado de no dejarse llevar por el cúmulo de conocimientos y novedades, sobre todo si éstos se ejercen desde una base no fundamentada en las virtudes y los valores.

Por lo tanto, cuando la sociedad cambia es necesario un programa educativo integral a efecto de lograr que las nuevas generaciones se formen como líderes con sensibilidad social y comprometidos para hacer el bien, bajo la premisa de los valores y el conocimiento, con directrices de una conciencia basada en el amor al prójimo. Se requiere establecer procedimientos que aseguren una adecuada selección de personas con características de liderazgo positivo, para desempeñar funciones, sobre todo en nombramientos trascendentes en todos los niveles de la política y de profesionales para la iniciativa privada, ya que aún existen algunas con mucha ética moral.

\section{La Responsabilidad Social en el contexto educativo}

En el marco de esas ideas, las instituciones educativas deben concebirse como una organización que funciona en su sociedad y genera impactos, tanto sobre las personas que laboran en ella, como sobre su entorno social y natural. Respecto de lo planteado surge la posición de Flórez (2005) quien comenta que:

... La Responsabilidad Social es una instancia que pretende solidificar las bases individuales intentando responder críticamente a la ambivalencia de valores y prioridades en los distintos estratos sociales por medio de una educación enfocada en el individuo y sus necesidades humanas. La responsabilidad social universitaria hace un llamado a las instituciones de la enseñanza superior a reconstruir y reelaborar la cultura y el saber para desarrollar una sociedad más solidaria y humana. En definitiva, será de suma importancia la unificación de voluntades y conciencias, ser entes proactivos los cuales generen realidades concretas... (p. 56)

Seguidamente, basados en la importancia que en la actualidad expresa la Responsabilidad Social, además de reiterar su vínculo académico, se desarrollarán indicadores implícitos con la gestión de los líderes que la dirigen.

Las instituciones educativas tienen la misión de formar ciudadanos con un perfil de emprendedores sociales, independientemente del área del conocimiento 
donde se desenvuelvan. Para Gómez (2019), el emprendimiento social está orientado a identificar y abordar problemáticas sociales en las comunidades. estilo de liderazgo transformacional de los líderes educativos favorece este tipo de emprendimiento con un alto sentido de Responsabilidad Social.

Un elemento a dimensionar se centra básicamente en la necesidad de concretar a través de la presente investigación, la labor que se ha venido generando en instituciones educativas a nivel de Responsabilidad Social (RS) en su entorno, y la visión que tiene precisamente ese entorno respecto de esa labor. Se entiende primeramente por percepción, aquella estructura significante propia del ser humano, es decir, el hombre observa todo aquello que desea observar de manera selectiva (Martínez, 2005).

Sin embargo, al agregársele el término entorno y aludiendo la posición del autor citado, la percepción del entorno viene a ser la estructura significante de un grupo de seres pensantes que están involucrados directamente con un sector específico, con el fin de recoger información para evaluarla y generar criterios, los cuales podrán ser usados de manera positiva o negativa.

Existen investigaciones en torno al liderazgo en las organizaciones educativas; es el caso de Horn y Marfan (2010), quienes hicieron una recopilación de evidencias sobre la relación entre ambos aspectos.

Desde esa óptica, la investigación se propuso como objetivo analizar el liderazgo transformacional como herramienta para la consolidación de la responsabilidad social en Instituciones de educación del Municipio de San Juan del Cesar de la Guajira, específicamente la Institución Educativa Manuel Antonio Dávila, la Institución Educativa María Emma Mendoza, Institución Educativa María Auxiliadora y la Institución Educativa José Eduardo Guerra.

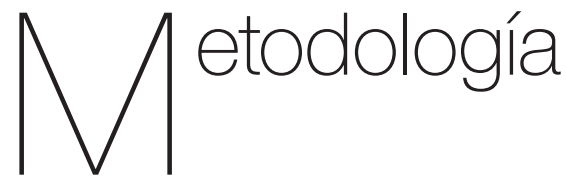

Para efectos de este estudio, la investigación asumió la posición epistemológica del conocimiento científico, ubicada en el enfoque cuantitativo, desde el punto de vista filosófico, centrado en el paradigma positivista. Al respecto, Cook y Reichardt (1995), citados por Hurtado (2010), consideran que "el paradigma cuantitativo, posee una concepción positivista, hipotético-deductiva, particularista, con énfasis en la objetividad, orientada a los resultados y propia de las ciencias naturales" ( $p$. 5). Los mencionados autores consideran que frecuentemente los investigadores que siguen este tipo de paradigma, se interesan solo por descubrir, identificar o verificar relaciones de causa-efecto, partiendo de diversas teorías.

Sin embargo, es necesario destacar que lo cuantitativo se refiere a la recolección y análisis de los datos que, para esta investigación en particular, usó como técnica la observación, definida por Méndez (2011) como "el proceso donde se perciben ciertos rasgos existentes en la realidad por medio de un esquema previo de lo que se quiere investigar" (p. 96). 
Desde esta perspectiva, se utilizó un diseño de campo, donde se requirió del uso de la técnica mediante encuesta estructurada, con la finalidad de obtener datos pertinentes de las fuentes de información. Esta técnica permitió conocer en realidad, las condiciones de los institutos educativos en relación al desarrollo o aplicación de las dimensiones e indicadores, los cuales conforman las variables objeto de estudio (Liderazgo transformacional y Responsabilidad social).

Con respecto a la técnica de observación mediante encuesta, Méndez (2011) la define como aquella que "permite el conocimiento de las motivaciones, las actitudes y las opiniones de los individuos con relación a su objeto de investigación" (p. 155). Es por ello que esta técnica brindó la oportunidad a la investigadora de conocer lo que realmente piensan las fuentes primarias de información (directoresdocentes y miembros de la comunidad), para determinar, con los datos recabados, el logro de los objetivos formulados.

Para la recolección de datos, se diseñaron tres (3) cuestionarios: El primero para medir la variable Liderazgo transformacional, conformado por 38 ítems, y dirigido a docentes y directivos de las instituciones educativas. El segundo orientado a medir la variable Responsabilidad social, de 20 ítems, y dirigido igualmente a docentes y directivos de las instituciones. Un tercer cuestionario para medir Responsabilidad social (de 20 ítems), fue aplicado a miembros de las comunidades que conforman el entorno de las instituciones educativas.

Los tres instrumentos se realizaron con una escala de cinco opciones de respuesta, a saber: Siempre, casi siempre, a veces, casi nunca y nunca. Estos cuestionarios, antes de ser aplicados, fueron sometidos a las correspondientes pruebas de validez y confiabilidad, arrojando valores altos para ambos criterios.

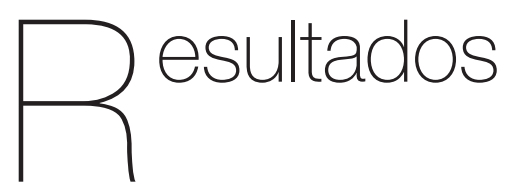

Partiendo de la aplicación de los instrumentos de recolección de información y el procesamiento estadístico de los datos obtenidos, se obtuvieron los siguientes resultados para las variables en estudio, sus dimensiones e indicadores. Con respecto al Liderazgo transformacional, las dimensiones estuvieron identificadas como Valores que caracterizan el liderazgo y los factores que integran el liderazgo transformacional. El instrumento fue aplicado a los docentes y directivos de las instituciones en estudio, y se llegó a los siguientes resultados, expresados en la Tabla 1.

Tabla 1

Variable: Liderazgo Transformacional

\begin{tabular}{lcccc}
\hline \multirow{2}{*}{ Dimensiones } & \multicolumn{2}{c}{ Docentes } & \multicolumn{2}{c}{ Directores } \\
\cline { 2 - 4 } & Promedio & Desviación & Promedio & Desviación \\
\hline Valores que caracterizan el liderazgo transformacional & 3.30 & .50 & 3.26 & .50 \\
Factores que integran el liderazgo transformacional & 3.26 & .46 & 3.30 & .50 \\
Promedio general & 3.28 & .48 & 3.28 & .50 \\
\hline
\end{tabular}


La variable Liderazgo transformacional arrojó un promedio para la respuesta de los encuestados de 3.28, con una muy baja dispersión de las mismas (.50), lo que permite ubicarlas dentro de la categoría alta. En este sentido, afirman los directores que, dentro de su ejercicio, consideran los valores que caracterizan al liderazgo transformacional y se ejecutan los factores que lo integran, logrando ejercer un ejercicio cónsono a los requerimientos de la institución.

En cuanto a la opinión de los docentes, se evidencia en sus respuestas un promedio de 3.28, con una muy baja dispersión de las mismas (.48), lo que lleva a ubicarlas dentro de la categoría moderada, a diferencia de lo expresado por los directores. Así, señalan los docentes que los directores ejecutan de forma moderada los valores que caracterizan el liderazgo y los factores que lo integran.

Al respecto de la respuesta de los encuestados, es necesario destacar lo expresado por Robbins y Judge (2015), quienes consideran al liderazgo transformacional como la integración de los elementos esenciales de la dirección, capaces de explicar, tanto el perfil del gerente como su inherencia en el desempeño laboral. Esto se traduce en una posición de mando o poder, que debe producir una sinergia o cooperación entre el líder y sus colaboradores.

De igual manera, puede inferirse que los resultados alcanzados también coinciden con los planteamientos de Flórez (2005), quien expresa que el liderazgo constituye un elemento fundamental para las instituciones educativas, donde se debe integrar cada una de las funciones de la docencia, brindando la posibilidad a los que ejercen esta labor de ejecutar sus actividades de acuerdo a los requerimientos institucionales.

Con respecto a la variable Responsabilidad social (RS), después de la recogida y procesamiento de la información recolectada de la aplicación de los cuestionarios a las tres poblaciones (directores, docentes y comunidades), se llegó al siguiente resultado (ver Tabla 2).

Tabla 2

Variable: Responsabilidad Social

\begin{tabular}{lcccccc}
\hline \multirow{2}{*}{ Dimensiones } & \multicolumn{2}{c}{ Docentes } & \multicolumn{2}{c}{ Directores } & \multicolumn{2}{c}{ Comunidades } \\
\cline { 2 - 7 } & Promedio & Desviación & Promedio & Desviación & Promedio & Desviación \\
\hline $\begin{array}{l}\text { Percepción del entorno respecto } \\
\text { a la responsabilidad social }\end{array}$ & 3.19 & .50 & 3.68 & .57 & 3.10 & .55 \\
\hline $\begin{array}{l}\text { Estrategias para el ejercicio de } \\
\text { la responsabilidad social }\end{array}$ & 3.17 & .51 & 3.81 & .59 & 3.19 & .60 \\
\hline \begin{tabular}{l} 
Promedio general \\
\hline
\end{tabular} & 3.18 & 0.51 & 3.74 & .58 & 3.14 & .57 \\
\hline
\end{tabular}

Partiendo de los resultados de la variable Responsabilidad social (RS), las respuestas de los docentes fue posible ubicarlas dentro de la categoría moderada, con un promedio de 3.18 y una desviación de .51. En este sentido, señalan 
los encuestados que se posee una percepción, en este mismo nivel, del entorno en el cual se desenvuelven y de las estrategias que se ejecutan vinculadas con el ejercicio de la responsabilidad social.

Atendiendo al resultado anterior, para Guerrero et al. (2014), la responsabilidad social es "un modelo de trabajo y organización que permite retribuir a la sociedad lo que la organización toma de ella, es una forma de hacer negocios de manera sustentable, es tener una sólida visión" (p. 18). Esta definición evidencia la importancia de invertir en el desarrollo educativo de la comunidad, por cuanto si lo hace en condiciones favorables, el entorno del cual depende cada persona podrá seguir generando beneficios para la misma.

Con relación a la respuesta de los directores, se obtuvo un promedio de 3.74 y una desviación de .58, ubicadas en la categoría alta. De acuerdo a esto, los directores consideran que en ese mismo nivel poseen conocimiento acerca de la RS e implementan estrategias para ejercerla en el entorno en el cual se desenvuelven las instituciones educativas a las cuales representan.

Por otra parte, las comunidades expresaron con un promedio 3.14 y una desviación de .57 (categoría moderada), que las instituciones educativas (representadas por docente y directores en el caso de este estudio), ejercen de forma moderada la RS que tienen con el entorno que las rodea.

Según los resultados anteriores, es necesario destacar que la RS se define como el compromiso de las organizaciones por lograr un desarrollo sostenible; se trata de un equilibrio entre crecimiento económico, impacto ambiental y bienestar social. Por ello, la implementación de la RS tiene grandes retos para las instituciones educativas, con relación a cada una de las dimensiones que la conforman.

En lo que respecta a la dimensión económica de la RS, existe el reto de mantener competitivas a las instituciones con el fin de satisfacer requerimientos cada vez más exigentes, tanto de los clientes internos como externos. De esta manera, se estará contribuyendo con el desarrollo social sostenido.

En cuanto a la dimensión ambiental, el desafío reside, entre otros, en la reducción significativa de emisiones al aire, desechos tóxicos y aguas residuales y la optimización en el uso de los recursos, involucrando desde la escuela la realización de campañas, la concienciación de los actores y el desarrollo de sus valores de compromiso.

Por su parte, la dimensión social tiene dos perspectivas, una interna y otra externa. En la perspectiva interna la institución persigue crear espacios de trabajo enriquecedores con igualdad de oportunidades y condiciones laborales seguras y saludables. A través de esto, la institución se asegura del cumplimiento con la legislación nacional e internacional y se propicia el ejercicio de valores éticos. En la perspectiva externa, la institución podrá establecer una comunicación con los grupos de interés (comunidad, gobierno, bancos, proveedores) y busca formas de mejorar la interrelación con estos.

El llevar a la práctica la RS requiere de personas visionarias, determinadas, con altas convicciones morales y capaces de generar compromiso por parte de sus 
colaboradores. Por lo tanto, se necesitan líderes organizacionales con características de transformacionales.

En ese orden de ideas, los líderes deberán tener la capacidad de imaginar un futuro, de visualizar un estado para su institución, plantearse situaciones de forma diferente, descubrir alternativas innovadoras y definir nuevas formas de hacer el trabajo. Deberán, de igual manera, poseer habilidades para comunicar y generar compromisos entre sus colaboradores, que conduzcan eficazmente hacia el logro de los objetivos planteados.

Así mismo, el líder sustentará su visión en valores y generará compromisos por medio de su ejercicio; modelará, motivará, tendrá la habilidad para ver lo mejor de la gente y colocarla en la posición adecuada; dedicarán tiempo y realizarán acciones para conocer a su gente, habilidades, capacidades y aspiraciones. A partir de dicho conocimiento, lograrán asignar tareas y ubicar a sus colaboradores en posiciones que resalten sus fortalezas, permitiéndoles así generar sentimientos de confianza y logro. Esto le ayudará a crear ambientes de trabajo enriquecedores y sentimientos de pertenencia a la organización.

Aunado a lo expresado, los líderes serán personas con determinación, coraje, y disciplina, tenaces, disciplinados y exigentes. A los líderes los mueve una gran determinación, una gran fuerza interna a la que podría llamarse coraje y que le permite afrontar problemas, levantarse cuantas veces caigan y continuar hacia el alcance de la visión propuesta. Tendrán igualmente habilidad para identificar y preparar sucesores.
La preocupación del líder por la institución va más allá de su estadía en su puesto. Es por ello que, cuando el momento del retiro llega, se esforzará por identificar y formar a la persona que asegurará la continuidad del trabajo. Los líderes son humildes, no requieren adulaciones. Poseen un interés sincero por su trabajo, por su crecimiento, por conducir al alcance de grandes resultados. Su deseo no es llegar a ser importantes, ni que se les reconozca como importantes, simplemente desean que su institución sea grandiosa. Siempre verán en otros la razón del éxito y encontrarán en sí mismos las razones del fracaso, además de que están dispuestos a cambiar, a mejorar. Se autoevalúan, se definen metas personales, se consideran a sí mismos en proceso de aprendizaje.

Seguidamente, la $\mathrm{RS}$ requiere igualmente de líderes organizacionales y sociales para su implementación, ya que son personas que, por sus características, tienen la capacidad de visualizar trabajos sostenibles, ofertar servicios con un valor agregado ambiental y social, crear ambientes de trabajo enriquecedores, donde los colaboradores se sientan motivados y valorados; ser exigentes con sus proveedores no sólo en términos de calidad sino también en el ejercicio de la $\mathrm{RS}$, de colaborar con la comunidad en actividades de beneficio mutuo y obtener beneficios atractivos. Solamente los líderes están preocupados por el éxito de sus instituciones, así como por el bienestar de la sociedad en general.

Para precisar orientaciones estratégicas generales de RS, es provechoso enfocar dos (2) líneas de acción institucional: 
1. En lo que concierne a la gestión interna: La meta es orientarla hacia la transformación de la institución educativa en un pequeña comunidad ejemplar de democracia, equidad (supresión de las segregaciones y corrección de los privilegios), transparencia (política y económica), y hacer de ella un modelo de desarrollo sostenible (política de protección del medio ambiente, uso de papel reciclado, tratamiento de los desechos).

2. En cuanto a la docencia: La meta es la de capacitar a los docentes en el enfoque de la Responsabilidad Social y promover en cada una de las especialidades, el aprendizaje basado en proyectos de carácter social, abriendo el salón de clase hacia la comunidad como fuente de enseñanza significativa y práctica aplicada a la solución de problemas reales.

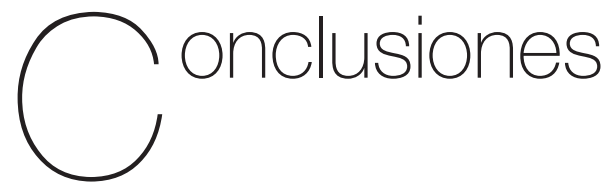

La importancia del Liderazgo Transformacional en el contexto educativo radica en el hecho de que, a pesar de que son visiones y objetivos diferentes a los del contexto gerencial, la gestión también se caracteriza por la necesidad de planificar, organizar, dirigir y controlar los procesos de la labor educativa. Actualmente se busca que las instituciones se involucren en los procesos de cambio que la sociedad del conocimiento está exigiendo.
La responsabilidad social de las instituciones educativas también requiere líderes sensibilizados hacia la realidad social de su entorno y el líder cumple un rol fundamental para facilitar este proceso.

El trabajo efectuado, contextualizado en la población objeto de estudio (docentes, directores y comunidades), evidenció que, aunque se ejecuta un liderazgo transformacional con un nivel moderado, el mismo debe evaluarse y ajustarse a los requerimientos de las instituciones analizadas, por cuanto es necesario que se integren todos los elementos que involucran a la RS, con el fin de que las instituciones alcancen los objetivos establecidos y las comunidades reciban el apoyo que requieren de las mismas.

Es imperativo, entonces, que las instituciones educativas logren abrir espacios a través de la información y del conocimiento, para que los estudiantes aprendan a tomar decisiones autónomas y responsables, con respecto a ellos mismos, su entorno y su comunidad. Estos conocimientos, actitudes, destrezas y comportamientos, fomentados por las instituciones educativas con relación a la responsabilidad social, deben estar inspirados en los valores y principios fundamentales, coherentes con los valores y principios constitucionales.

De igual manera, será necesario promover desde las instituciones educativas, la formación en valores de los empleados y sus respectivas familias, guardando el máximo respeto a sus creencias. Con ello, podrá visualizarse en el país, empresas promotoras para la creación de grupos de apoyo a los ciudadanos, a sus instituciones, y a sus diversas comunidades. 
El sistema educativo colombiano tiene que fomentar en el país el desarrollo de la sociedad, concertando con las partes involucradas, las internas y las externas, la idea de promover la RS como un compromiso, identificando y comprendiendo los efectos de sus acciones y la noción de los impactos que genera. Deberá, además, desarrollar compromiso para concertar y responder con las partes interesadas; compromiso para rendir cuentas de las decisiones, la transparencia de las acciones y dar respuestas a los problemas que se presentan en la sociedad, cumpliendo con lo declarado y pactado en su misión y visión. En este sentido, deberán hacerse conscientes las instituciones educativas, que, a diferencia de las empresas, el estudiante no es un cliente que compra un producto, sino que constituye el producto final.

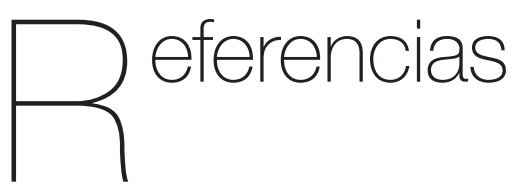

Arroyo, R. (2017). Habilidades Gerenciales. Desarrollo de destrezas, competencias y actitud. Colombia: Ediciones ECOE.

Drucker, P. (1998). Working knowledge: How organizations manage what they know. Boston: Harvard Business SchoolPress.

Dubrir, A. (1913). El liderazgo en Evolución. Informe general de Líderes. Venezuela: IESA.

Fajardo, K., Zutta, D., Caicedo, E. \& Luna, E.G. (2018). Variables Asociadas al manejo de la Autoridad en Docentes. Informes Psicológicos, 18(1), 113-132.

Flórez, E. (2005). Elementos de La Ética, Filosofía, Política y Derecho. Venezuela: Editorial Los Libros de "El Nacional".

Gómez, M. (2019). La Mentalidad emprendedora. Colombia: Ediciones de la U.

Guerrero, P., DuBrin, A., García, E., Quiñones, W., \& Alers, C. (2008). Relaciones humanas: comportamiento humano en el trabajo. México: Pearson Educación.

Horn, A. \& Marfan, J. (2010). Relación entre liderazgo educativo y desempeño escolar: Revisión de la investigación en Chile, 9(2), 82-104. Recuperado de https://scielo. conicyt.cl/pdf/psicop/v9n2/art05.pdf

Hurtado, J. (2010). Guía para la comprensión holística de la ciencia. Tercera Edición, Caracas: Fundación Sypal.

Maestres, J. (2016). Los Lideres Transformadores. Venezuela: IESA.

Martínez, E. (2005). La responsabilidad social como manifestación del desempeño ético gerencial en cadenas de farmacias. (Tesis de grado), Universidad Dr. Rafael Belloso Chacin (URBE), Maracaibo. Zulia. Venezuela

Méndez, C. (2011). Metodología. Diseño y desarrollo del proceso de investigación con énfasis en ciencias empresariales. México: Editorial Limusa, S.A.

Piñango, L. (2012). Liderazgo para la Gente. Informe IESA Venezuela. Recuperado de http://www.debatesiesa.com/ 
Porter, M., \& Kramer, M. (2002). The competitive advantage of corporate philanthropy. USA: Harvard Business Review.

Robbins, S., \& Judge, T. (2015). Comportamiento organizacional. México: Pearson

Salazar,M.(2006). ElLiderazgoTransformacional ¿Modelo para organizaciones educativas que aprenden?, Unirevista, 1(3), 1-12. Recuperado de http://www.tutores. escasto.ipn.mx/mariogerardoricardo/ file s / 2012 / 02 / LIDER A Z G O TRANSFORMACIONAL-EJEMPLO4.pdf
Suárez, E., (2019). Liderazgo transformacional y productividad en los docentes de la Escuela Fiscal Antonio de Sucre de la provincia de Guayas Ecuador. Recuperado de http://cybertesis.unmsm.edu.pe/ handle/cybertesis/10476 